\title{
PATRONES DE DIVERSIDAD DE EPÍFITAS EN BOSQUES DE TIERRAS BAJAS Y SUBANDINOS
}

\author{
Epiphytic diversity patterns in lowland and subandean forests
}

Palabras clave: Araceae, bosque húmedo subandino, bosque húmedo tropical, Bromeliaceae, hostilidad ambiental, Orchidaceae, riqueza de especies.

Key words: Araceae, subandean cloud forest, tropical rain forest, Bromeliaceae, environmental hostility, Orchidaceae, species richness.

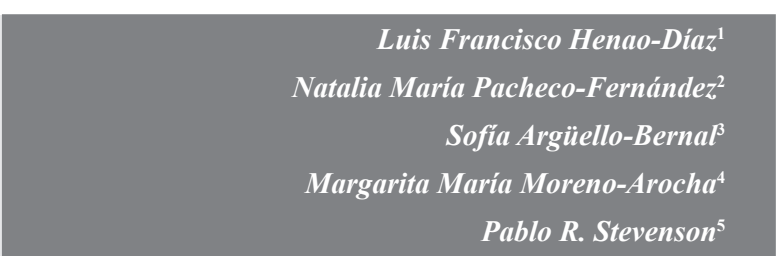

ABSTRACT

Tropical rainforests are the most diverse ecosystems on the planet. Although many theories have been proposed to explain their richness we currently know little about their relative importance. This diversity is threatened by anthropogenic disturbances such as fragmentation and forest degradation. In this study we compared epiphytic plant diversity patterns in lowland and subandean forests. More epiphytic diversity was found in subandean (PNN Cueva de los Guácharos) compared to lowland forest (Palmarí Reserve). In addition a higher abundance of epiphytes was found in conserved forest. Tree host diversity was higher in lowland forests than in subandean forests, so we can reject the possibility that differences between the patterns of epiphytic diversity are caused by regional effects of plant diversity. Our results suggest that humidity seems to be the most important environmental filter explaining epiphyte diversity. parece ser el filtro ambiental más importante para explicar la variación en diversidad de epífitas.

\footnotetext{
Universidad de los Andes. Bogotá, Colombia.1f.henao28@uniandes.edu.co. Autor para correspondencia

Universidad de los Andes. Bogotá, Colombia.nm.pacheco27@uniandes.edu.co

Universidad de los Andes. Bogotá, Colombia.1s.arguello75@uniandes.edu.co

Universidad de los Andes. Bogotá, Colombia. margaritamma@gmail.com

Universidad de los Andes. Bogotá, Colombia. pstevens@uniandes.edu.co
} 


\section{INTRODUCCIÓN}

Los bosques tropicales subandinos se diferencian de los de tierras bajas en estructura y funcionamiento; estos últimos están caracterizados por una alta productividad y reciclaje de nutrientes en el ambiente (Bruijnzeel \& Veneklaas, 1998). A pesar de que la diversidad de árboles en los bosques tropicales subandinos tiende a ser mayor que en los de tierras bajas y disminuye paulatinamente con la altitud (Gentry, 1988), aún no conocemos plenamente cuales son los factores que más afectan estos patrones de diversidad. En medio de ella, los epífitos constituyen una parte importante de estos bosques andinos, con más del 30\% de la flora vascular (Distler et al., 2009). Estas aproximaciones utilizan todas las especies de la flora, no exclusivamente árboles, para sugerir que los patrones para otros tipos de hábitos de crecimiento pueden ser diferentes a los descritos para flora arbórea (Jiménez et al., 2009). De hecho, se estima que el $10 \%$ de las plantas vasculares y cerca del $30 \%$ de la diversidad de plantas de bosques andinos están compuestas por epífitas (Gentry \& Dodson, 1987; Wolf \& Flamenco, 2003). Asimismo, en términos funcionales, las epífitas tienen un papel preponderante en la biomasa y reciclaje de nutrientes en los bosques de las tierras altas (Hofstede et al., 1993), en donde juegan un papel ecológico importante para otros niveles tróficos. Esto se debe a que las plantas epífitas proporcionan recursos tales como sitios de alimentación, refugio y reproducción para una gran variedad de organismos como anfibios e insectos (Wester et al., 2011).

Actualmente una de las hipótesis que explica los patrones de diversidad de plantas es la de hostilidad del ambiente, pues se ha favorecido la idea de que los patrones de diversidad conocidos son, en buena parte, explicados por efectos azarosos (Hubbell, 2001). Cuando hay filtros ambientales fuertes es de esperar una baja diversidad en ecosistemas donde las condiciones ambientales sean poco favorables. En el caso particular de las plantas epífitas, numerosos estudios han mostrado que son organismos muy susceptibles a desecación, por lo cual estas plantas son más abundantes en ambientes con condiciones altas de humedad (Gentry \& Dodson, 1987; Gentry, 1988). Según esta teoría, se predeciría que bosques alto andinos, considerados muy húmedos por la presencia de nubosidad constante (Melo \& Vargas, 2003), deberían albergar una alta diversidad de epifitos. El tipo de vida de estas plantas implica una fuerte dependencia a condiciones atmosféricas muy específicas; por ejemplo, sus abundantes requerimientos de agua les hacen altamente susceptibles a las sequías, producto de deforestación y pastoreo (Nöske et al., 2008). Estas características hacen de las briofitas y las epífitas, en general, plantas clave en la identificación de las alteraciones del bosque húmedo tropical (Hietz, 1999).

La presencia humana constituye un elemento determinante en la gravedad de fenómenos altamente amenazantes, como son la deforestación y la fragmentación de hábitat; con esto se demuestra la influencia de briofitas epífitas en la continuidad del bosque, pues ellas necesitan un área extensa para persistir (Zartman \& Nascimiento, 2006). De igual manera, es necesario mencionar que la fragmentación incrementa el riesgo de extinción de poblaciones locales (Hietz, 1999). Esto se manifiesta a través de la disminución de tamaños poblacionales como consecuencia de la reducción en el hábitat, el consecuente aumento en el efecto de borde (Hietz-Seifert et al., 1996) y la disminución del potencial de migración de las especies (Hietz, 1999). Estudios recientes demuestran el impacto que ejercen los bordes establecidos por los asentamientos humanos en la destrucción del bosque húmedo tropical (Laurance et al., 2002; Laurance et al., 2007). Por ejemplo, Hietz (1999) describe el efecto de borde como una de las formas más frecuentes de alteración del ecosistema asociadas a deforestación. Otras alteraciones pueden ser las quemas para agricultura, la conversión de bosque para cría de ganados, la tala selectiva y los monocultivos de especies arbóreas exóticas.

Por otro lado, el patrón de distribución de las especies de forofitos se encuentra relacionado con el historial de disturbios o variación edáfica, fisiografía, profundidad del suelo, textura, contenido de nutrientes, drenaje, vientos y apertura del dosel 
(Gentry, 1988). Por lo tanto, cuando hay límites de la distribución, uno de los ecotonos más definidos se da entre los suelos periódicamente inundados y los de tierra firme (Sloan, 1987). También se ha documentado una asociación entre la distribución de especies y patrones medioambientales como la topografía y la variación del tipo de suelo. En algunas ocasiones es difícil la detección de patrones existentes o la influencia de variables ambientales en la estructura de la vegetación (Hubbell, 2001). Sin embargo, en otros casos no existen asociaciones consistentes con variaciones ambientales, por esto aparentemente están relacionadas con la historia de regeneración local de las especies (Sloan, 1987).

El presente estudio pretende comparar la diversidad de plantas epífitas entre el bosque húmedo tropical y subandino bajo diferentes condiciones de conservación. Según la hipótesis de hostilidad ambiental, se esperaría que bosques de várzea cercanos a un río presenten mayor diversidad de epífitas que bosques de tierra firme con mayor variación en la humedad. Del mismo modo es de esperarse que bosques subandinos con mayor disponibilidad hídrica posean mayor diversidad que los de tierras bajas. Adicionalmente, dado que la colonización de epífitas sobre forofitos es un proceso que ocurre progresivamente a través del tiempo, se esperaría que bosques secundarios tuvieran menor diversidad de epífitas que bosques prístinos o secundarios tardios. Finalmente, se evalúa si se presenta especificidad de epífitas por forofitos particulares y si las diferencias encontradas se pueden atribuir a efectos regionales que afecten la diversidad local, a partir de la diversidad de los forofitos estudiados en dos zonas de muestreo.

\section{MATERIALES Y MÉTODOS}

\section{ÁREA DE ESTUDIO}

El primer lugar de estudio fue la reserva natural Palmarí ubicada sobre el río Javarí en el Estado de Amazonas en Brasil, a $35 \mathrm{~km}$ de la desembocadura de este río sobre el Amazonas; posee un área aproximada de 40 ha de bosques primarios, bosques sucesionales tardios y unas pocas zonas de bosques secundarios tempranos. La reserva se encuentra a $91 \mathrm{~m}$ de altitud y cubre ecosistemas tanto de tierra firme, como de várzea. El Javarí es un río de aguas intermedias comunicado con varias lagunas y canales; es, además, un cauce de aguas estacionales cuyo nivel varía aproximadamente $18 \mathrm{~m}_{\text {año }}{ }^{-1}$, por ello la zona de várzea suele ser extensa (Samper, 2003). El nivel más bajo del río se alcanza aproximadamente en septiembre, $\mathrm{y}$ a partir de ese momento aumenta gradualmente hasta mayo, cuando alcanza su nivel más alto, la temperatura máxima y mínima a la sombra es de $34^{\circ} \mathrm{C}$ y $20^{\circ} \mathrm{C}$, respectivamente, mientras que la temperatura máxima y mínima a cielo abierto es de $48^{\circ} \mathrm{C}$ y $26^{\circ} \mathrm{C}$, respectivamente. El promedio aproximado de lluvia es de $3400 \mathrm{~mm} \mathrm{año}^{-1}$ y su humedad es del $92 \%$. La temporada de lluvias ocurre entre enero y marzo, y la temporada seca entre junio y septiembre (RNP, 2010).

El segundo lugar de estudio corresponde a los bosques subandinos del parque nacional natural (PNN) Cueva de los Guácharos. El parque se ubica al sur del departamento del Huila y norte del Caquetá, en los municipios de Acevedo y San José del Fragua, respectivamente. Se caracteriza por la presencia de cañones y laberintos, además de las formaciones cavernosas producidas por acción química y mecánica del río Suaza y sus afluentes. Este parque presenta un gradiente altitudinal entre los 1650 y $2800 \mathrm{~m}$ de altitud, con una temperatura anual promedio de $16^{\circ} \mathrm{C}$, una precipitación media anual de $3100 \mathrm{~mm}$ y un $87 \%$ de humedad relativa. La temporada de lluvias se extiende desde abril a noviembre y la época menos lluviosa de diciembre a marzo. La red hidrográfica y la zona amortiguadora pertenecen a las cuencas del Magdalena (ríos Suaza y Guarapas) y Caquetá (Caño Agachado y la quebrada La Fraguosa; UPNN, 2005).

\section{MUESTREO DE EPÍFITAS Y FOROFITOS}

En la reserva natural Palmarí se realizaron cinco transectos, cada uno con una distancia máxima de $100 \mathrm{~m}$. Los transectos uno y dos se encuentran sobre bosque maduro, el tercero se encuentra en bosque secundario, mientras que los transectos cuatro y cinco se realizaron sobre el plano de inundación del río Javarí. 
Asimismo, en el PNN Cueva de los Guácharos se realizaron cuatro transectos (distanciados entre sí $100 \mathrm{~m}$, como máximo). Dispuestos de la siguiente forma; el uno y cuatro se realizaron sobre bosque secundario, mientras los transectos dos y tres en bosque primario.

Los transectos fueron escogidos de tal forma que su inicio ocurriera cerca $(5-15 \mathrm{~m})$ del borde de un disturbio antrópico que generara un claro en el bosque de al menos una hectárea o un borde natural (la orilla de un río). A lo largo de cada transecto se utilizó el método de punto cuadrante cada $25 \mathrm{~m}$, estableciendo cuatro cuadrantes por punto. Para cada cuadrante se escogió el forofito más próximo al punto, que tuviera un diámetro entre 25 y $60 \mathrm{~cm}$.

Para cada uno de los forofitos muestreados se registró su distancia lineal al transecto, el CAP (circunferencia a la altura del pecho) y su categoría taxonómica; así mismo, se cuantificó la cantidad e identidad de epífitas vasculares asociadas que no fueran lianas. Se registró la altura y para estandarizar solo se tuvieron en cuenta las epífitas presentes a una altura menor o igual a $12 \mathrm{~m}$. Para estas epífitas se registró categoría taxonómica y presencia o ausencia de conexión con el suelo.

\section{ANÁLISIS DE DATOS}

El análisis de datos se desarrolló considerando el punto como unidad de muestreo (excepto para los análisis de especificidad de epífitas, en los que los individuos fueron las unidades de muestreo). Así pues, se promedió la cantidad de individuos por morfotipo, género y familia de los forofitos de los cuadrantes, y se calculó el error estándar por punto; el anterior procedimiento se realizó para cada uno de los puntos de los transectos a sus respectivas distancias. No se encontraron diferencias significativas en los promedios de individuos de epífitas por puntos para morfotipos, géneros y familias en ninguna de las dos locaciones, por tanto, se utilizaron los datos de los morfotipos para los análisis posteriores. Del mismo modo, se calculó el índice de diversidad de Shannon para cada punto por transecto. Los puntos fueron divididos según el tipo de bosque en cada una de las localidades $\mathrm{y}$, posteriormente, fueron aleatorizados 1000 veces sin remplazo para corregir por esfuerzo de muestreo usando EstimateS (Colwell, 2009). El anterior análisis se realizó para las epífitas y los forofitos. Tambien se probó la normalidad del promedio de morfotipos y del promedio del índice de Shannon por transecto (R Core Development Team, 2010).

En términos descriptivos, se buscaron patrones altitudinales de abundancia en género y familia en los forofitos; del mismo modo se calculó la abundancia de forofitos por género en el muestreo, sus dimensiones y el número de forofitos para cada distancia en el transecto. Adicionalmente se exploró una posible relación entre categorías de CAP en intervalos de $10 \mathrm{~cm}$ y la cantidad de epífitas por morfotipo, género y familia. Se realizó una ordenación basada en la composición de las epífitas para varios forofitos de la misma especie, con el fin de examinar la existencia de especificidad en las epífitas que se encuentran en cada especie de planta, de forma que si ocurre especificidad, las especies de forofitos se agruparan en la ordenación.

\section{RESULTADOS}

\section{RESERVA NATURAL PALMARÍ}

\section{Epífitas}

Se encontraron 423 individuos epífitos correspondientes a 89 morfotipos, 96 géneros y 26 familias. Las familias de epífitas más abundantes fueron Araceae (79.6\%), Pteridaceae (3\%) y Marcgraviaceae $(1.75 \%)$. La familia Araceae fue la única que presentó un marcado patrón altitudinal en abundancia, lo cual evidencia una tendencia negativa con respecto a la altura de los forofitos (Figura 1a). Asimismo, se hallaron diferencias significativas entre la riqueza de epífitas entre transectos (ANOVA $F=14.4, p<0.001$; Figura $2 a$ ). Consistente con lo esperado, el transecto de bosque secundario joven (no. 3) fue el que presentó los valores más bajos de riqueza de epífitas, con respecto a los transectos de otros tipos de bosques y uno bosque de plano de inundación (no 4) que mostró el mayor valor. Por su parte, el análisis de rarefacción reveló diferencias entre los tipos de 
bosque: el bosque primario mostró un promedio de 44 especies, el inundable 38 y el secundario 12 (Figura 3).

El índice de diversidad de Shannon muestra una significancia estadística a través de los transectos $(F=3.15, p=0.036)$. La diferencia se encuentra entre el transecto 2 de bosque primario y el 3 de bosque secundario (Tukey HSD $p=0.037$ ).

\section{Forofitos}

Se registraron un total de 94 forofitos pertenecientes a 29 familias (Figura 4). El mayor número de forofitos se encuentra en las familias Fabaceae $(18.09 \%)$, Urticaceae $(9.57 \%)$, Euphorbiaceae $(8.51 \%)$, Myristicaceae $(8.51 \%)$ y Moraceae (6.38\%; Tabla 1).
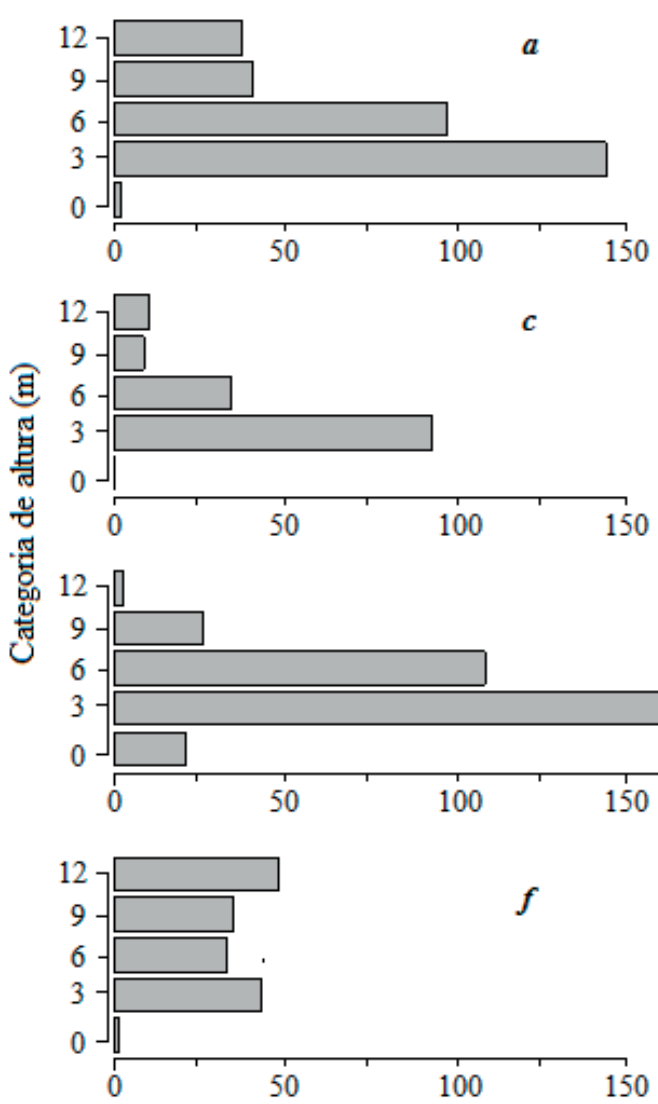

Número de epifitas

Figura 1. Número de epífitas por categoría de altura. Reserva natural Palmarí familia: $\boldsymbol{a}$. Araceae. PNN Cueva de Los Guácharos familias: $\boldsymbol{b}$. Araceae, $\boldsymbol{c}$. Polypodiaceae, $\boldsymbol{d}$. Dennstaedtiaceae, $\boldsymbol{e}$. Piperaceae, $\boldsymbol{f}$. Bromeliaceae, $\boldsymbol{g}$. Orchidiaceae 


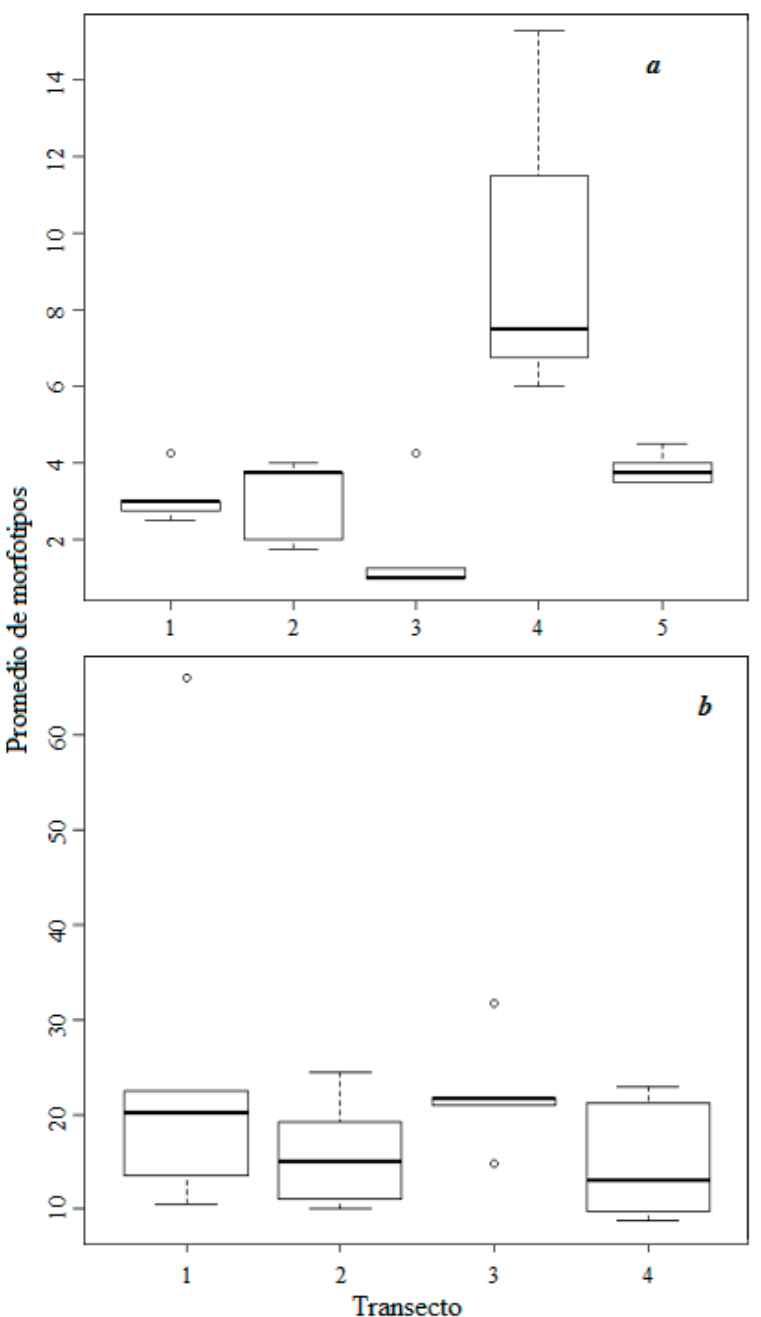

Figura 2. Promedio de morfotipos de epífitas por transecto $\boldsymbol{a}$. Reserva natural Palmarí: transectos $1 \mathrm{y}$ 2 bosque maduro, 3 bosque secundario, 4 y 5 bosque inundable; $\boldsymbol{b}$. PNN Cueva de los Guácharos: transectos 1 y 4 bosque secundario, 2 y 3 bosque primario

en el número de epífitas con la altura (Figura 1e). Las familias Bromeliaceae y Orchidiaceae enseñan una disposición bimodal, en la cual hay una disminución en el número de epífitas en las alturas intermedias (Figura 1f-g).

Para los transectos no se encontraron diferencias significativas en la riqueza promedio de los morfotipos (Kruskal-Wallis $K=2.91, p=0.41$; Figura $2 b$ ). Asimismo, el análisis de rarefacción mostró que el bosque secundario contiene un total de 97 especies frente a 72 del bosque primario (Figura 3). El índice de diversidad de Shannon presenta diferencias estadísticas entre transectos

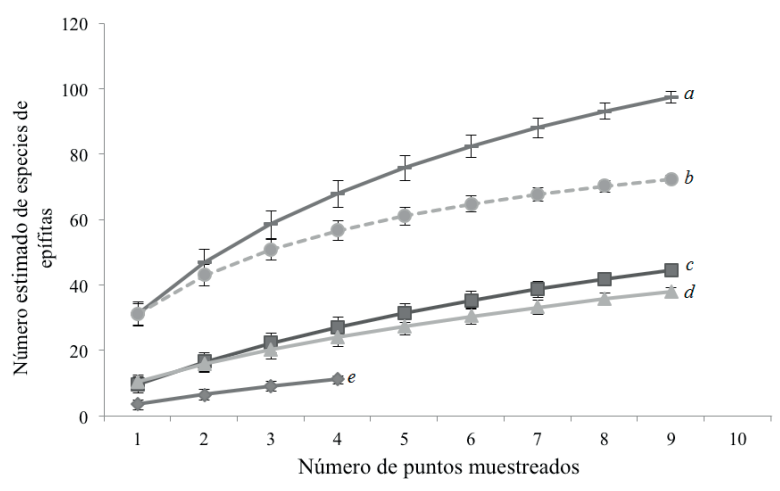

Figura 3. Número acumulado de especies a medida que se realizaron más puntos de muestreo para cada tipo de bosque en las dos localidades. Las barras muestran la desviación estandar. $\boldsymbol{a}$. bosque secundario Guácharos, $\boldsymbol{b}$. bosque primario Guácharos, $c$. bosque primario Palmarí, $\boldsymbol{d}$. bosque indundable Palmarí, $\boldsymbol{e}$. bosque Secundario Palmarí

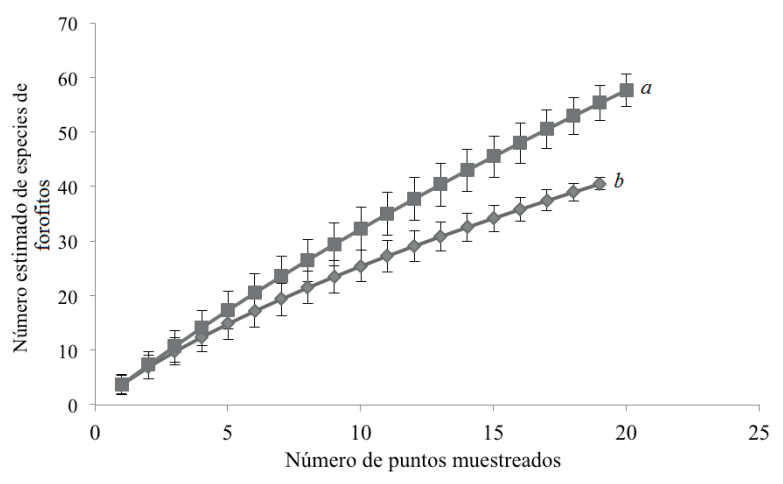

Figura 4. Número acumulado de especies de forofitos a medida que se acumularon más puntos de muestreo. Las barras muestran la desviación estandar. $\boldsymbol{a}$. reserva natural Palmarí, $\boldsymbol{b}$. PNN Cueva de los Guácharos

Tabla 1. Porcentaje de las principales familias en forofitos reserva natural Palmarí y PNN Cueva de los Guácharos

\begin{tabular}{clr}
\hline Área de estudio & Familia & Porcentaje \\
\hline & Fabaceae & 18.09 \\
& Cecropiaceae & 9.57 \\
Reserva natural & Euphorbiaceae & 8.51 \\
Palmarí & Myristicaceae & 8.51 \\
& Moraceae & 6.38 \\
& Malpighiaceae & 5.32 \\
& Juglandaceae & 17.95 \\
PNN Cueva de & Euphorbiaceae & 15.38 \\
los Guácharos & Lauraceae & 14.10 \\
& Moraceae & 11.54 \\
& Malvaceae & 7.69 \\
& Cunoniaceae & 3.85 \\
\hline
\end{tabular}


$(F=3.85, \mathrm{p}=0.03)$; esta significancia se encuentra entre el transecto 1 de bosque secundario y 3 de bosque primario $(p=0.03)$.

\section{Forofitos}

Se registraron un total de 79 forofitos pertenecientes a 21 familias (Figura 4). El mayor número de forofitos pertenece a las familias Juglandaceae $(18.0 \%)$, Euphorbiaceae $(15.4 \%)$, Lauraceae (14.1\%) y Moraceae (11.5\%; Tabla 1). El análisis discriminante no encontró agrupación en los forofitos por la especificidad de sus epífitas. Esto ocurrió tanto en la reserva natural Palmarí como en el PNN Cueva de los Guácharos (resultados no mostrados).

\section{DISCUSIÓN}

\section{DISTRIBUCIÓN DE EPÍFITAS}

Se encontró aproximadamente $26 \%$ más de individuos, así como una mayor diversidad de especies de epífitas en el PNN Cueva de los Guácharos que en la reserva natural Palmarí. Esto se puede evidenciar en la riqueza de especies (Figura 3) y en mayores índices de diversidad de Shannon, lo cual indica que el bosque subandino tiene mayor diversidad de epífitas respecto al bosque amazónico. Esto podría deberse a la disponibilidad de agua, un recurso limitante en el crecimiento de epífitas, así como a la exposición directa a la luz (van Reenen \& Gradstein, 1983; Gentry \& Dodson, 1987; Benzing, 1990; Castro-Hernandez et al., 1999; Kessler, 2001; Kromer et al., 2005; Gradstein, 2008). En este sentido, se ha observado recurrentemente que la riqueza de epífitas en ambientes montanos está influenciada por la humedad del aire (Ingram et al., 1996; Nieder et al., 1999; Kreft et al., 2004), donde algunas de las fuentes que favorecerían la humedad ambiental serían la alta precipitación y la lluvia horizontal por condensación (Vogelmann, 1973; Stadtmuller, 1987; Obregon et al., 2011).

El papel de la evapotranspiración también se ha discutido, pues esta es mayor en tierras bajas por su mayor temperatura, lo cual limitaría la disponibilidad de agua y aumentaría el riesgo de desecación para los epífitas (Benzing, 1990; Zotz \& Hietz, 2001). Adicionalmente se ha postulado que las diferencias topográficas producen un dosel heterogéneo y discontinuo en tierras altas, lo cual permitiría mayor penetración de la luz a los estratos intermedios del bosque en donde las epífitas podrían instalarse (Melo \& Vargas, 2003). Para las bromelias y orquídeas se encontró una distribución bimodal, dado que algunos géneros de estas familias tienen adaptaciones que les permiten evitar o reducir la desecación frente a condiciones de alta irradiación solar. Es posible que las agrupaciones de estos, en alturas diferentes del forofito, generen la distribución bimodal observada (Cascante-Marin et al., 2006).

Las familias de las epífitas más abundantes encontradas en este trabajo concuerdan con las descritas por estudios anteriores en los cuales se sitúan preferentemente las orquídeas, aráceas, pteridofitos y bromelias (Küper et al., 2004; Larrea \& Werner, 2010); sin embargo, las abundancias relativas de estas cambian drásticamente entre las localidades. En el bosque amazónico se presenta una mayor dominancia por la familia Araceae, mientras que en el PNN Cueva de los Guácharos no se presentan familias dominantes, lo cual concuerda con lo descrito por Leimbeck \& Balslev (2001) para bosques húmedos neotropicales. Por ejemplo, la dominancia de las aráceas en tierras bajas es de $79.6 \%$, mientras en tierras altas ocupan el tercer rango de abundancia (10.2\%). Adicionalmente, es de esperar que este tipo de patrones varíen de acuerdo al rango de altura en el cual se evalúe el forofito, que en nuestro caso fue entre cero y doce metros. Posiblemente, la inclusión de estratos más altos hubiese generado una mayor representación de especies de epífitas más típicas del dosel, como Orchidaceae (Cascante-Marin et al., 2006).

Los patrones de abundancia de las familias Araceae, Polypodiaceae, Dennstaedtiaceae y Piperaceae en los forofitos son decrecientes con la altura. Esto puede deberse a la dificultad que tienen las epífitas para escalar los forofitos desde los estratos bajos del bosque. También puede ocurrir por 
la necesidad de mantenerse conectadas al suelo donde la disponibilidad de agua es mayor, o de mantener una altura lo suficientemente alejada del dosel para evitar la exposición directa a la luz solar y disminuir con ello el riesgo de desecación (Gradstein, 2008). Por el contrario, las bromelias y orquídeas se encuentran en todas las alturas de los forofitos sin una altura preferente; esto se debe a la independencia hídrica de estas plantas con respecto al suelo, así como a posibles adaptaciones a condiciones de desecación.

Las diferencias en los promedios de epífitas entre los transectos están determinadas por los tipos de bosque en cada una de las localidades. En el caso de la reserva natural Palmarí, la mayor riqueza de especies se encuentra en el bosque primario, lo cual puede deberse al grado de conservación del bosque (Wolf et al., 2009), donde las epífitas han tenido mayor tiempo para establecerse. El bosque inundable contiene una riqueza similar con respecto al bosque primario, lo que se esperaría por su estado de conservación. Adicionalmente se puede ver que los bosques inundables poseen en promedio más epífitas que los demás bosques (Figura 2a); esto podría explicarse por la humedad que el río Javarí genera sobre estos terrenos. El transecto realizado sobre el bosque secundario muestra la menor riqueza de especies debido al menor tiempo de colonización. Además, se ha mencionado que la estructura abierta del dosel, donde la humedad del aire es menor y hay mayor irradiación, deja como resultado solo unas pocas especies tolerantes a estas condiciones (Kromer et al., 2005).

Por su parte, el PNN Cueva de los Guácharos muestra una diferencia clara entre los tipos de bosque, pues el bosque secundario contiene 25 especies más que el bosque primario (Figura $2 b$ ). Este hallazgo resulta contrario a lo esperado, ya que se ha documentado que la regeneración de comunidades epífitas en ambientes intervenidos es muy lenta (Gradstein, 2008). Sin embargo, este fenómeno puede ser explicado por el forrajeo de los micos churucos que habitan en el parque, quienes prefieren moverse y alimentarse en los bosques primarios. El forrajeo es usualmente destructivo, pues los micos escarban, arrancan y deshojan las epífitas en busca de invertebrados, lo cual podría afectar la abundancia en este tipo de bosque.

\section{FOROFITOS}

Se encontró una mayor diversidad de forofitos en el bosque amazónico comparado con el bosque de tierras altas (Figura 4). Asimismo, se observa una predominancia de Fabaceas en bosques amazónicos, lo cual concuerda con lo descrito por Cascante-Marin et al. (2006) para bosques maduros o con muy poca intervención. Sin embargo, no se observó la misma tendencia para el PNN Cueva de los Guácharos, donde predominan otras familias, entre las que se incluyen algunas exclusivas de montañas (e.g. Juglandaceae) (Cascante-Marin et al., 2006).

La relación entre diversidad de epifitas y de forofitos para las localidades en estudio es inversa, puesto que se encontró una mayor diversidad de forofitos en Palmarí, pero una menor diversidad de epifitas; mientras que en el PNN Cueva de los Guacharos se encontró una menor diversidad de forofitos, pero una mayor diversidad de epifitas. Teniendo esto en cuenta, estudios anteriores han encontrado que el forofito desempeña un papel importante en la determinación de la distribución de las epífitas y, por tanto, en la existencia de especies particulares de árboles importantes, los cuales varían de acuerdo a propiedades físicas y riqueza de nutrientes (Castro-Hernandez et al., 1999). No se encontró especificidad en las epífitas de los forofitos. Además, los pocos estudios llevados a cabo en este tema muestran poco consenso al respecto. Por una parte, se ha encontrado poca especificidad (Hietz, 1997; Laube \& Zotz, 2006; Vergara-Torres et al., 2010), y cuando esta se observa, ha sido atribuida a las características de la superficie de los forofitos, relacionadas con la retención de agua o características arquitectónicas de este (Callaway et al., 2002; Merwin et al., 2003; Gradstein, 2008).

En los resultados obtenidos en este estudio se observa la existencia de una mayor relación entre la diversidad de epífitas y las condiciones ambien- 
tales, pues su abundancia es mayor en el bosque subandino donde las condiciones son más favorables para ellas. Adicionalmente, en los bosques de tierras bajas, quienes poseen la mayor riqueza de epífitas son los planos de inundación al borde del río, con esto se sugiere que la abundancia de epífitas tiene una mayor relación con el filtro ambiental. Esto concuerda con lo descrito por Gradstein (2008), quien afirma que dada la sensibilidad de las epífitas a cambios en las condiciones atmosféricas, pequeños cambios en el clima local pueden generar fuertes impactos en estas comunidades. Adicionalmente, y teniendo en cuenta que los ciclos de inundación y sequía en el Amazonas son muy marcados, es posible que el microclima de estos bosques cambie drásticamente en poco tiempo, de esta manera se dificultaría el establecimiento de una mayor diversidad de epífitas. Esto llevaría a pensar que cambios climáticos que originen periodos más largos de sequía o inundación generarían, a su vez, cambios irreversibles en las comunidades epífitas ya establecidas.

\section{CONCLUSIONES}

Se encontró una mayor diversidad de epífitas en el bosque de tierras altas. Tambien, se observó que en bosques de tierras bajas e inundables hay una marcada predominancia de Araceae, mientras que en bosques de tierras altas la diversidad está representada por Piperaceae, Bromeliaceae y Araceae. Es posible que las diferencias de diversidad estén relacionadas con la humedad disponible, lo cual favorece los bosques de tierras altas con mayor disponibilidad de recursos y condiciones atmosféricas adecuadas en el ámbito local.

\section{REFERENCIAS BIBLIOGRÁFICAS}

Benzing, D.H. (1990). Vascular epiphytes: general biology and related biota. Cambridge: Cambridge University Press. 354 p.

Bruijnzeel, L.A., \& Veneklaas, E.J. (1998). Climatic conditions and tropical montane forest productivity: The fog has not lifted yet. Ecological Society of America, 79(1), 3-9.
Callaway, R., Reinhart, K., Moore, G., Moore, D., \& Pennings, S. (2002). Epiphyte host preferences and host traits: mechanisms for species-specific interactions. Oecologia, 132(2), 221-230.

Cascante-Marín, A., Wolf, J., Oostermeijer, J., den Nijs, J., Sanahuja, O., \& DuránApuy, A. (2006). Epihpytic bromeliad communities in secondary and mature forest in a tropical premontane area. Basic and Applied Ecology, 7, 520-532.

Castro-Hernández, J.C., Wolf, J.H.D., GarciaFranco, J.G., \& González-Espinosa, M. (1999). The influence of humidity, nutrients and light on the establishment of the epiphytic bromeliad Tillandsia guatemalensis in the highlands of Chiapas, Mexico. Revista de Biología Tropical, 47(4), 763-773.

Colwell, R.K. (2009). EstimateS: Statistical estimation of species richness and shared species from samples (Version 8.2). User's Guide and application. Recuperado el 10 de febrero de 2012 de http://purl.oclc.org/ estimates.

Distler, T., Jorgensen, P.M., Graham, A., Davidse, G., \& Jiménez, I. (2009). Determinants and prediction of broadscale plant richness across the western neotropics. Annals of the Missouri Botanical Garden, 96(3), 470-491.

Gentry, A.H., \& Dodson, C.H. (1987). Diversity and biogeography of neotropical vascular epiphytes. Annals of the Missouri Botanical Garden, 74(2), 205-233.

Gentry, A.H. (1988). Changes in plant community diversity and floristic composition on environmental and geographical gradients. Annals of the Missouri Botanical Garden, 75, 1-34. 
Gradstein, S. (2008). Epiphytes of the tropical montane forest - impact of deforestation and climate change. En: S.R. Gradstein, J. Homeier \& D. Gansert (eds.). The Tropical Mountain Forest, Patterns and Processes in Biodiversity Hotspot (pp.:5165). Göttingen: Göttingen Centre for Biodiversity and Ecology.

Hietz, P. (1997). Population dynamics of epiphytes in a mexican humid montane forest. Journal of Ecology, 85(6), 767-775.

Hietz, P. (1999). Diversity and conservation of epiphytes in a changing environment. IUPAC, 70(11), 1-11.

Hietz-Seifert, U., Hietz, P., \& Guevara. S. (1996). Epiphyte vegetation and diversity on remnant trees after forest clearance in southern Veracruz, Mexico. Biological Conservation, 75(2), 103-111.

Hofstede, R.G.M., Wolf, J.H.D., \& Benzing, D.H. (1993). Epiphytic mass and nutrient status of an upper montane rain forest. Selbyana, 14, 37-45.

Hubbell, S.P. (2001). The unified neutral theory of biodiversity and biogeography. Princeton: Princeton University Press. 375 p.

Ingram, A.U., Ferrell, S.W., Ingram, K., \& Nadkarni, N.M. (1996). Floristic composition of vascular epiphytes in a Neotropical cloud forest, Monteverde, Costa Rica. Selbyana, 17, 88-103.

Jimenez, I., Distler, T., \& Jorgensen, P.M. (2009). Estimated plant richness pattern across northwest South America provides similar support for the species-energy and spatial heterogeneity hypotheses. Ecography, 32(3), 433-448.

Kessler, M. (2001). Pteridophyte species richness in Andean forests in Bolivia. Biodiversity and Conservation, 10, 1473-1495.
Kreft, H., Koster, N., Kuper, W., Nieder, J., \& Barthlott, W. (2004). Diversity and biogeography of vascular epiphytes in Western Amazonia, Yasuní, Ecuador. Journal of Biogeography, 21, 1463-1476.

Kromer, T., Kessler, M., Gradstein, S., \& Acebey, A. (2005). Diversity patterns of vascular epiphytes along an elevational gradient in the Andes. Journal of Biogeography, 32, 1799-1809.

Küper, W., Kreft, H., Nieder, J., Köster, N., \& Barthlott, W. (2004). Large-scale diversity patterns of vascular epiphytes in Neotropical montane rain forests. Journal of Biogeography, 31(9), 1477-1487.

Larrea, M.L., \& Werner, F.A. (2010). Response of vascular epiphyte diversity to different land-use intensities in a neotropical montane wet forest. Forest Ecology and Management, 260(11), 1950-1955.

Laube, S., \& Zotz, G. (2006). Neither hostspecific nor random: vascular epiphytes on three tree species in a Panamanian lowland forest. Annals of Botany, 97(6), 1103-1114.

Laurance, W.F., Lovejoy, T.E., Vasconcelos, H.L., Bruna, E.M., Didham, R.K., Stouffer, P.C., Gascon, C., Bierregaard, R.O., Laurance, S.G., \& Sampaio, E. (2002). Ecosystem decay of Amazonian forest fragments: a 22-year investigation. Conservation Biology, 16(3), 605-618.

Laurance, W.F., Nascimento, H.E.M., Laurance, S.G., Andrade, A., Ewers, R., Harms, K.E., Luizão, R.C., \& Ribeiro, J. (2007). Habitat fragmentation, variable edge effects, and the landscape-divergence hypothesis. PLoS ONE, 2(10), e1017.

Leimbeck, R.M., \& Balslev, H. (2001). Species richness and abundance of epiphytic Araceae on adjacent floodplain and upland 
forest in Amazonian Ecuador. Biodiversity and Conservation, 10(9), 1579-1593.

Melo, A., \& Vargas, R. (2003). Evaluación ecológica y silvicultural de ecosistemas boscosos, Colombia. Ibagué: Universidad del Tolima, CRQ, CARDER, CORPOCALDAS, CORTOLIMA. 235 p.

Nieder, J., Engwald, S., \& Barthlott, W. (1999). Patterns of neotropical epiphyte diversity. Selbyana, 20, 66-75.

Merwin, M.C., Rentmeester, S.A., \& Nadkarni, N.M. (2003). The influence of host tree species on the distribution of epiphytic Bromeliads in experimental monospecific plantations, La Selva, Costa Rica. Biotropica, 35(1), 37-47.

Nöske, N., Hilt, N., Werner, F., Briehmen, G., Fridler, K., \& Sipman, H. (2008). Disturbance efects of diversity of epiphytes and moths in a montane forest in Ecuador. Basic and Applied Ecology, 9(1), 4-12.

Obregon, A., Gehrig-Downie, C., Gradstein, S.R., Rollenbeck, R., \& Bendix, J. (2011). Canopy level fog occurrence in a tropical lowland forest of French Guiana as a prerequisite for high epiphyte diversity. Agricultural and Forest Meteorology, 151(3), 290-300.

RNP [Reserva Natural Palmarí]. (2010). Reserva Natural Palmarí. Recuperado el 10 de febrero de 2012 de http://www.palmari. org/.

R Core Development Team. (2010). R: A Language and Environment for Statistical Computing. Vienna: R Foundation for Statistical Computing. ISBN: 3-90005107-0, recuperado de http://www.R-project. org.

Samper, T. (2003). Uso de un parche de Costus arabicus por parte de la comunidad de colibríes de la Reserva Natural Palmarí (Tesis de pregrado, Biología). Bogotá: Universidad de los Andes. 59 p.

Sloan, J. (1987). Tropical rainforest gap and trees species diversity. Annual Review of Ecology and Systematics, 18, 431-451.

Stadtmuller, T. (1987). Cloud forests in the humid tropics. A bibliographic review. Tokyo: United Nations University Press. 81 p.

UPNN [Unidad de Parques Nacionales Naturales]. (2005). Plan de Manejo 2005 - 2009 Parque Nacional Natural Cueva de los Guácharos. Bogotá: UPNN. 196 p.

van Reenen, G.B.A., \& Gradstein, S.R. (1983). Studies on colombian cryptogams XX. A transect analysis of the bryophyte vegetation along an altitudinal gradient on the Sierra Nevada de Santa Marta, Colombia. Acta Botánica Neerlandesa, 32, 163-175.

Vergara-Torres, C.A., Pacheco-Álvarez, M.C., \& Flores-Palacios, A. (2010). Host preference and host limitation of vascular epiphytes in a tropical dry forest of central Mexico. Journal of Tropical Ecology, 26(6), 563-570.

Vogelmann, H.W. (1973). Fog precipitation in the cloud forests of eastern Mexico. Bioscience, 23, 96-100.

Wester, S., Mendieta-Leiva, G., Nauheimer, L., Wanek, W., Kreft, H., \& Zotz, G. (2011). Physiological diversity and biogeography of vascular epiphytes at Río Changuinola, Panama. Flora - Morphology, Distribution, Functional Ecology of Plants, 206(1), 6679.

Wolf, J.H.D., \& Flamenco, S.A. (2003). Patterns richness and distribution of vascular epiphytes in Chiapas, Mexico. Journal of Biogeography, 30, 1689-1707. 
Wolf, J.H.D., Gradstein, S.R., \& Nadkarni, N.M. (2009). A protocol for sampling vascular epiphyte richness and abundance. Journal of Tropical Ecology, 25(02), 107-121.

Zartman, C., \& Nascimento, H. (2006). Are habitat-tracking metacommunities dispersal limited? Inferences from abundance-occu- pancy patterns of epiphylls in Amazonian forest fragments. Biological Conservation, 127(1), 46-54.

Zotz, G., \& Hietz, P. (2001). The physiological ecology of vascular epiphytes: current knowledge, open questions. Journal of Experimental Botany, 52, 2067-2078. 